\title{
A Novel Compact Antenna for Passive UHF RFID Tag Using $\pi$-Match and Meander Techniques
}

\author{
Younes EL HACHIMI ${ }^{1}$, Abdelmajid FARCHI ${ }^{2}$ \\ \{elhachimi.younes1@gmail.com ${ }^{1}$, abdelmajid.farchi1@ gmail.com ${ }^{2}$ \} \\ Laboratory of Engineering, Mechanics, Industrial Management and Innovation ${ }^{1,2}$ \\ University Hassan 1st, Faculty of Sciences and Technologies, Settat-Morocco
}

\begin{abstract}
In this work, a novel passive tag antenna has been designed, investigated and proposed for UHF RFID applications. It has a small size and can operate in the North and South American band (902-928 MHz). The proposed antenna was etched on a low-cost FR4 substrate with an overall size of $54 \times 24 \times 0.8 \mathrm{~mm}^{3}$ in order to be applied as UHF RFID tag antenna at the center frequency of $915 \mathrm{MHz}$. It consists of embedded $\Pi$-match, meandered arms, and an RFID chip placed at the center. The meander line technique is used to assure the miniaturization and the $\Pi$-match technique to have a good impedance matching. This antenna has a good reflection coefficient and good read range.
\end{abstract}

Keywords: Passive tag, UHF, RFID, small size, read range, meander line, П-match.

\section{Introduction}

In the previous years, and until today, considerable attention has been paid to radiofrequency identification (RFID) technology among researchers everywhere throughout the world attributable to its huge applications in logistics, retail management, electronic transport payment, access control, automotive security, automated libraries, healthcare industries, livestock management, etc. [1].

Radio frequency identification (RFID) is a technology for the contactless automatic identification. By using this technology, items can be recognized and followed. A RFID device is mainly made out of a reader, a tag that affixed to objects, and implanted computer system for data processing. In a passive back-dissipated RFID system, the reader transmits a modulated RF signal consistently every which way, and the tag, arranged inside the read-zone of the reader, receives the RF signal and generates a backscatter with recognizable proof information to the reader. The latter is used to peruse and write data from the tag remotely. The data processing system consists of a host computer and software to analyze the received data. Finally, the tag. It can be divided into two categories: active (with an onboard source of power, like a battery) or passive (without battery). Passive tag usually incorporates an antenna and an RF chip which has a memory to store distinguishing data [2]. The input impedance of the RFID chip must be conjugately matched with that of the antenna impedance to accomplish maximum most extreme power exchange [3]. In the available literature, several passive RFID tag antenna structures [49] have already been proposed.

There are four diverse frequency bands apportioned for RFID applications, low frequency (LF: 125-134 kHz), high frequency (HF: $13.56 \mathrm{MHz}$ ), ultra-high frequency (UHF: 860-960 
$\mathrm{MHz})$ [10] and microwave frequency $(2.45 \mathrm{GHz}$ and $5.8 \mathrm{GHz})$ [11]. Different nations have allocated different frequency bands for UHF-RFID applications; e.g., China (840-845 MHz), Europe (866-869 MHz), USA (902-928 MHz) and Australia (920-926 MHz). At LF and HF bands, the dimensions of the tag antenna are bigger since the wavelength is bigger, bringing about a small read range between the tag and the reader. The UHF tags have gathered more consideration as they offer longer read ranges, higher reading speeds, enable simultaneous detection of an increasing number of tags and need smaller antennas [12].

For handy applications, RFID tag antennas must be compact, suitable for low-cost and reasonable for ease large scale manufacturing [13] and must also have specific antenna impedance. Many papers presented in the literature provided different techniques for reducing the size of the antenna, such as meandering, fractal, inverted-F, or Split Ring Resonators (SRR) structures [14-17], and other for the impedance matching, such as T-match network [18], double T-matching structure [19] and inductively coupled loop [20].

In this paper, we propose a new tag antenna for UHF RFID applications which is intended to meet the requirements of reasonable compact size and good matching simultaneously. The proposed RFID antenna consists of Embedded П-match, meandered arms, and an RFID chip placed at the center. To have a good impedance matching between the antenna and the RFID chip, the technique of loading П-match is applied. For miniaturization, meander lines are used. This design has a simple structure with one layer of the dielectric. The performances of this RFID tag antenna supplemented with simulated results are likewise presented and discussed. It shows good read range characteristics throughout the UHF RFID band.

\section{Antenna design}

For the design, modeling, and simulation of the antenna, CST (Computer Simulation Technology) microwave studio [21], whose numerical analysis is based on the Finite Integration Technique was used. The geometry of the proposed tag antenna is depicted in Figure 1. It is printed on a FR4 substrate having a thickness of $0.8 \mathrm{~mm}$, dielectric constant of 4.4 and loss tangent of 0.025 . It is completely planar in nature. The proposed design is very simple and made out of an embedded П-match loaded at both ends with a couple of meandered arms. This tag is without a ground plane. The П-matching structure is used to satisfy the condition of the conjugate matching so as to obtain a good adaptation. Meander line technique helps in reducing the size of the antenna. The total dimension of the tag antenna is $54 \times 24 \times 0.8 \mathrm{~mm} 3$ which correspond to $(0.165 \lambda) \times(0.073 \lambda) \times(0.002 \lambda)$, where $\lambda$ is the free-space wavelength at $915 \mathrm{MHz}$. The optimized parameters are shown in Table 1. 


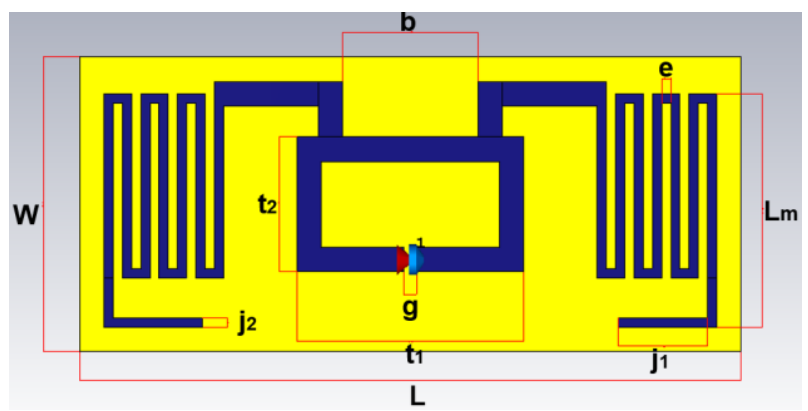

Fig. 1. Antenna structure.

Table 1. Dimensions of the proposed antenna.

\begin{tabular}{cccc}
\hline Parameters & Dimensions $(\mathrm{mm})$ & Parameters & Dimensions $(\mathrm{mm})$ \\
\hline $\mathrm{L}$ & 54 & $\mathrm{~d}$ & 11 \\
$\mathrm{~W}$ & 24 & $\mathrm{j} 1$ & 7.25 \\
$\mathrm{t} 1$ & 18.5 & $\mathrm{j} 2$ & 0.75 \\
$\mathrm{t} 2$ & 11 & $\mathrm{Lm}$ & 19 \\
$\mathrm{~g}$ & 1 & $\mathrm{e}$ & 0.75 \\
\hline
\end{tabular}

\section{Input impedance of the RFID chip}

The microchip used in UHF RFID tags have capacitive input impedance. In order to deliver the maximum power transfer, tag antennas are designed to have an inductive impedance. The input impedance of the antenna, Za should be complex conjugate of the chip impedance, $\mathrm{Zc}$ ( $\mathrm{Zc}$ $=\mathrm{Za}^{*}$ ). The chip used in this configuration is "Alien Higgs 4" [22]. It is a passive tag microchip having an input impedance of $\mathrm{Zc}=(18,43-\mathrm{j} 181,2) \Omega$ at $915 \mathrm{MHz}$ in North and South of America. Therefore, the antenna is designed for $\mathrm{Za}=(18,43+\mathrm{j} 181,2) \Omega$.

The RFID impedance can be modeled either by a parallel or series equivalent circuit $\mathrm{Rp}$, Cp, Rs, Cs. These parameters can be calculated as shown in (1) (2) (3) (4) [23]. In this work, we have modeled this microchip by a series equivalent circuit $(\mathrm{Rs}=18,43 \Omega, \mathrm{Cs}=0.955 \mathrm{pF}$ ). Figure 2 illustrates the modeled equivalent circuit. The parameters of the equations are as follows:

$$
\begin{aligned}
& \mathrm{R}_{\mathrm{p}}=\frac{\mathrm{Im}_{\text {chip }}{ }^{2}+\mathrm{R}_{\text {chip }}{ }^{2}}{\mathrm{R}_{\text {chip }}} . \\
& \mathrm{C}_{\mathrm{p}}=\frac{\mathrm{Im}_{\text {chip }}}{2 \pi \mathrm{f} .\left(\mathrm{Im}_{\text {chip }}{ }^{2}+\mathrm{R}_{\text {chip }}{ }^{2}\right)} . \\
& \mathrm{R}_{\mathrm{s}}=\mathrm{R}_{\text {chip }} . \\
& \mathrm{C}_{\mathrm{s}}=\frac{1}{2 \pi \mathrm{f} . \mathrm{Im}_{\text {chip }}} .
\end{aligned}
$$




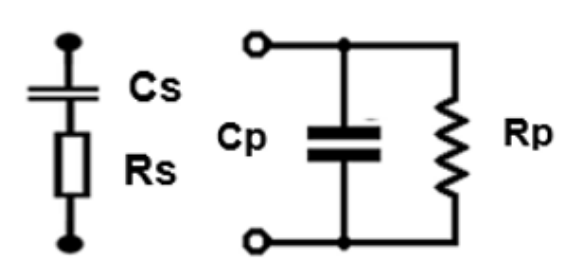

Fig. 2. Equivalent circuit of chip impedance

\section{Parametric study}

Since every geometrical parameter affects the antenna performance, a parametric study is being employed to optimize the final design. The radiating element has three key parameters: " $t 1$ ", the length of П-matching structure's rectangle, "b", the distance between the two arms of the П-matching structure, and " $\mathrm{j} 1$ ", the length of the end of meandered arms. Each physical attribute of the antenna is independently varied with all other parameters being kept unchanged. In order to examine the effect of the $\Pi$-match on the resistance and reactance of the tag, $t 1$ and $\mathrm{b}$ are varied and the result is presented in Figure 3 and Figure 4, respectively. It is seen from the graph that when the value of $\mathrm{t} 1$ is varied, the resistance values are slightly changed, and there is a significant change in the reactance values, i.e., a rise in parameter $t 1$ causes their magnitudes to rise ( important change for the imaginary part and a small one for the real part), while a fall in these parameters causes the magnitude to fall. For the distance $b$ between the two arms of the $\Pi$-matching structure, it can be seen that the change in $b$ causes a small change in the real and imaginary parts of the input impedance. The optimized values of $t 1=18.5 \mathrm{~mm}$ and $b=11 \mathrm{~mm}$, allowed us to have a good conjugate matching between the antenna and the chip. Figure 5 plotted the reflection coefficient of our RFID tag with different value's of $\mathrm{j} 1$ when $\mathrm{t} 2$ and $\mathrm{b}$ are fixed as $18.5 \mathrm{~mm}$ and $5 \mathrm{~mm}$ respectively. It is observed that the resonance frequency and the reflection coefficient decreases with the increase in $\mathrm{j} 1$. The best result was observed when the value of $\mathrm{j} 1$ was $7.25 \mathrm{~mm}$. We can conclude that the $\Pi$-matching structure has an effect on the impedance of the antenna and the length of meandered arms affects the resonance frequency.

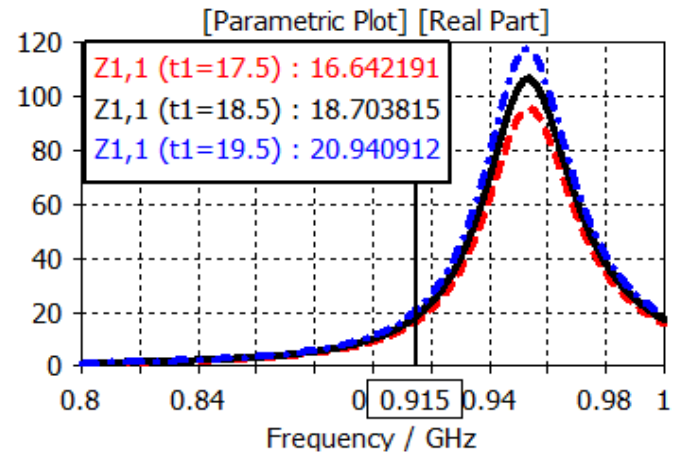

(a)

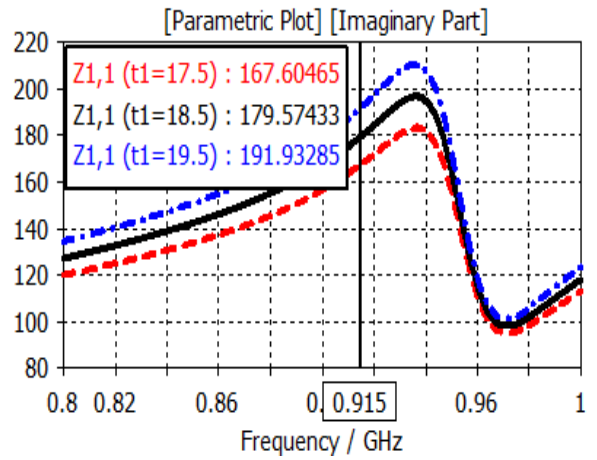

(b)

Fig. 3. Simulated input impedance of proposed antenna with various t1, (a) real part and (b) imaginary part. 
[Parametric Plot] [Real Part]

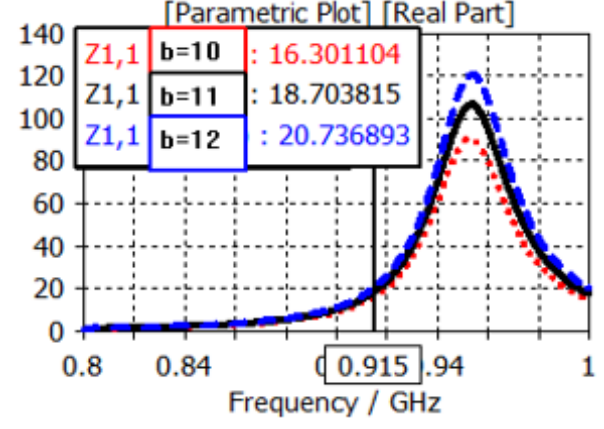

(a)

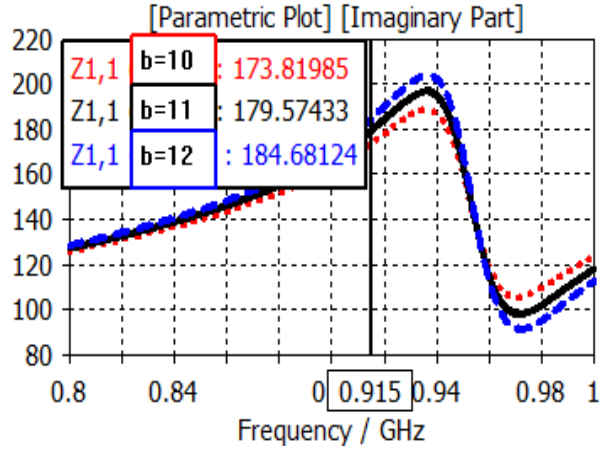

(b)

Fig. 4. Simulated input impedance of the proposed antenna, (a) real part and (b) imaginary part with various b

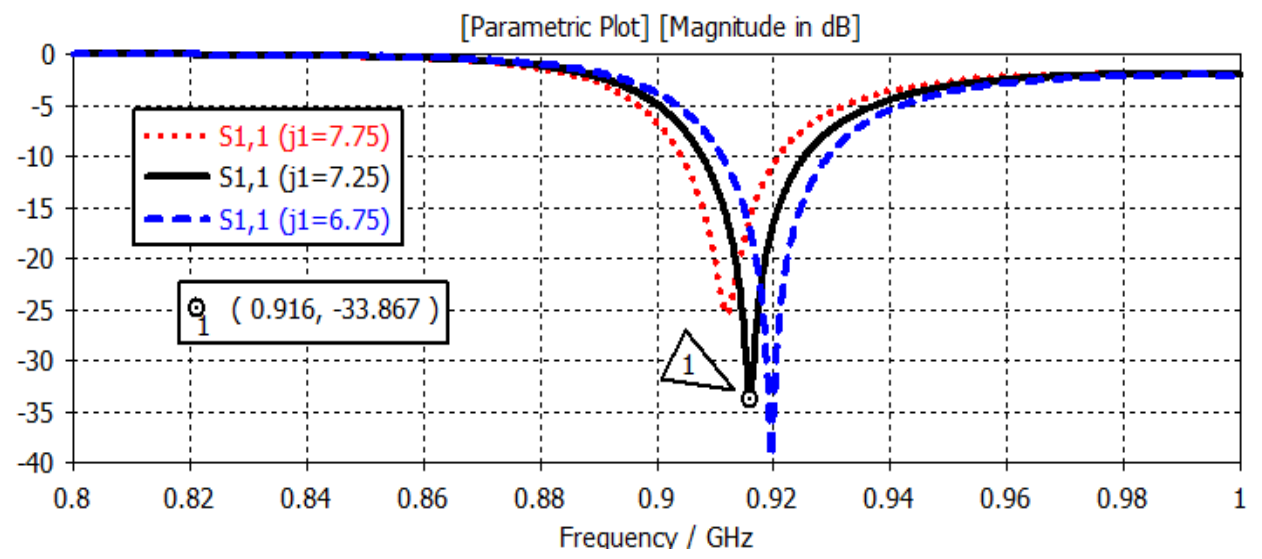

Fig. 5. Simulated return loss of the proposed antenna with various $\mathrm{j} 1$

\section{Results and discussion}

The goal of this study is to design a new tag antenna with compact and low profile structure for UHF-RFID band also a good return loss and matching between the tag antenna and the chip using the $\Pi$-matching structure and meander line technique. Figure 6 depicts the simulated reflection coefficient characteristics of the designed antenna as a function of frequency. We notice that the simulated return loss reaches a value of $-33.86 \mathrm{~dB}$ at $916 \mathrm{MHz}$ with a bandwidth of 17.61 MHz ranging from 907.79 to $925.4 \mathrm{MHz}$, almost covering the North American UHF RFID Band (902-928 MHz). This value of S11 is less than $-10 \mathrm{~dB}$, which reflects the antenna adaptation. The simulated real and imaginary part of the input impedance of the proposed tag antenna versus frequency is illustrated in Figure 7. As can be seen in the figure, the input impedance of the antenna is $(18.7+\mathrm{j} 179.57) \Omega$, and it is almost the value of complex conjugate of the chip impedance $(18,43-\mathrm{j} 181,2) \Omega$ at $915 \mathrm{MHz}$. This leads to the observation that this new shape (П) has allowed us to achieve a good conjugate matching between the antenna and the 
chip, which reduces the losses due to reflections. The simulated 3D gain of the proposed tag antenna is presented in Figure 8. We can notice that the gain reached a value of $1.31 \mathrm{~dB}$ at 915 MHz. The simulated 2D radiation pattern at E-plane and H-plane is shown in Figure 9. It can be observed that the radiation patterns are bidirectional in the E-plane and almost omnidirectional in the H-plane. To determine the performance of the tags, it is important to get the read range [3], which can be calculated utilizing the Friis-free space formula as:

$$
\mathrm{r}=\frac{\lambda}{4 \pi} \sqrt{\frac{\text { EIRP.G }_{\mathrm{r}} \cdot \tau}{\mathrm{P}_{\text {chip }}}}
$$

where $\lambda$ is the wavelength and EIRP is the effective isotropically radiated power, determined by local country regulations (e.g., $3.8 \mathrm{~W}$ in Europe and $4 \mathrm{~W}$ in the USA). $\mathrm{P}_{\text {chip }}$ is the minimum threshold power necessary to activate the RFID chip, $\mathrm{G}_{\mathrm{r}}$ is the gain of the receiving tag antenna, and $\tau$ is the power transmission coefficient. Hence, the calculated maximum read range was found to be $16.13 \mathrm{~m}$ at $915 \mathrm{MHz}$.

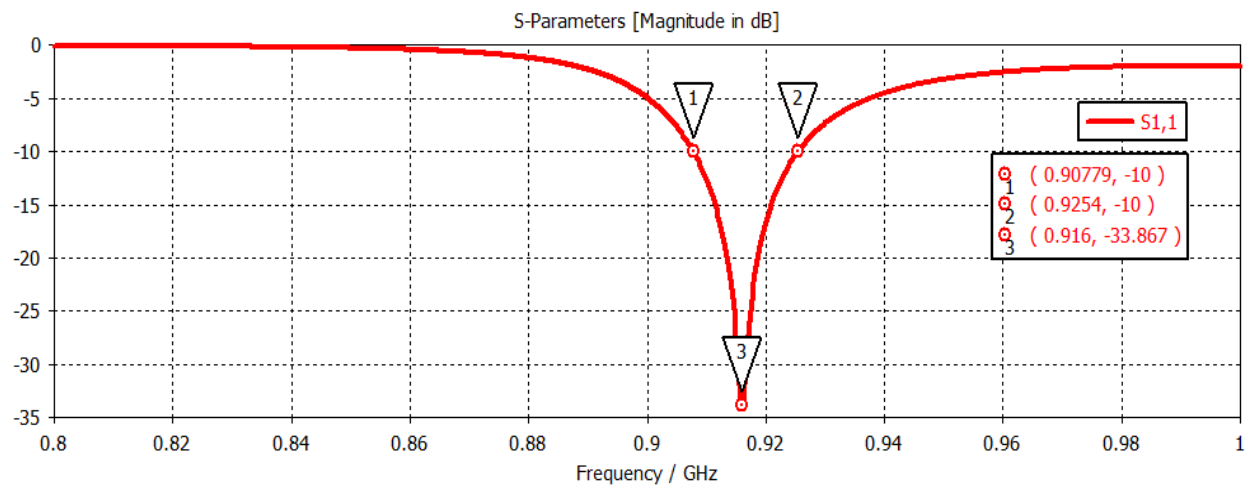

Fig. 6. Simulated return loss of the proposed tag antenna

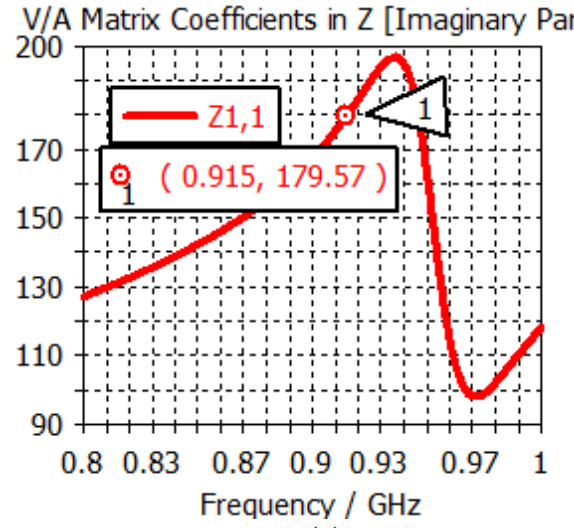

(a)

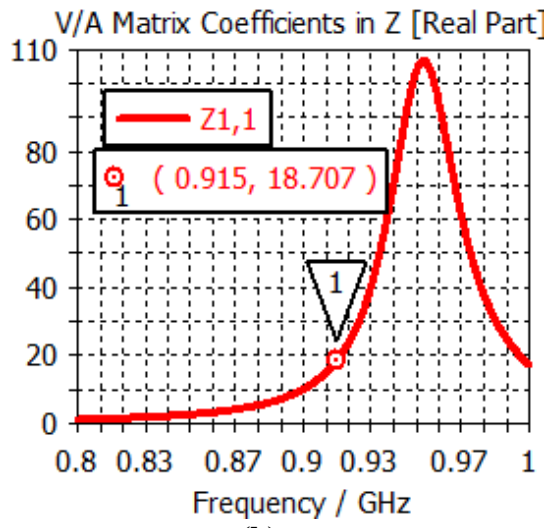

(b)

Fig. 7. Simulated Input impedance of the proposed antenna, (a) real part and (b) imaginary part. 


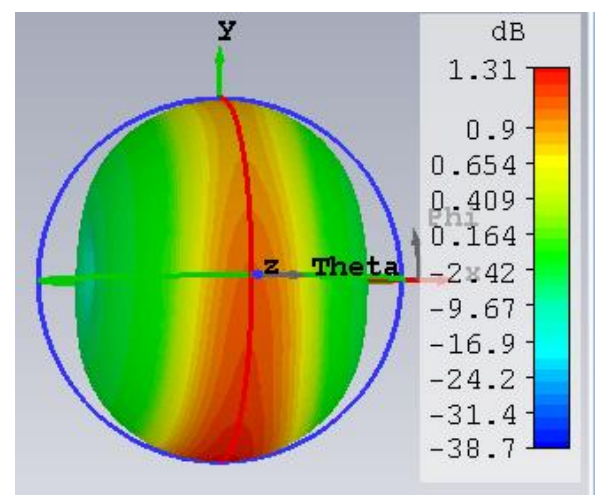

Fig. 8. Simulated 3D gain of the proposed tag antenna.

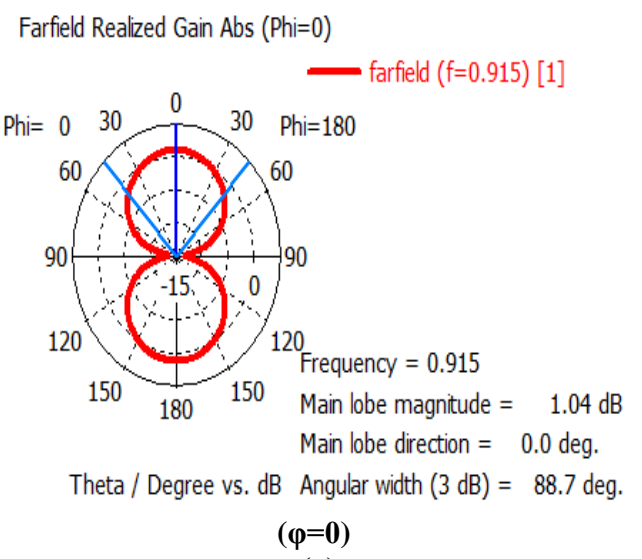

(a)

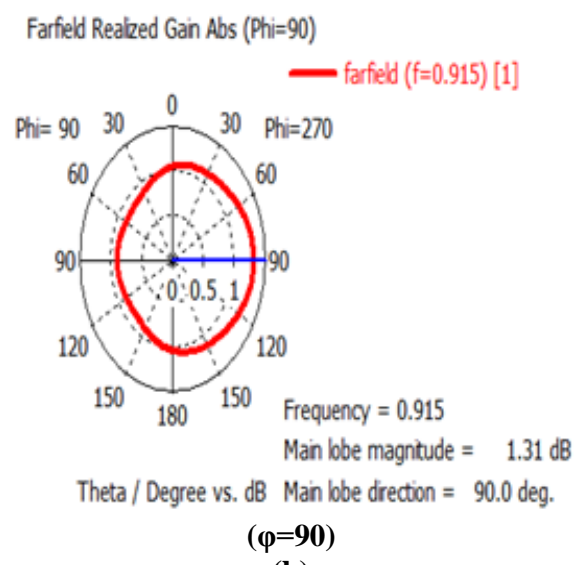

(b)

Fig. 9. Simulated 2D radiation pattern: (a) E-plane and (b) H-plane.

\section{Comparison}

In order to emphasize the importance of miniaturization, a comparison between the proposed tag with some other designed antennas in terms of volume, return loss, bandwidth, and read range is exhibited in Table 2, respectively. The first antenna [24] uses a semi-circle loop and two feeding strips and two triple-flipped meandered lines of symmetrical identity at both sides of the antenna. The second one [25] consists of meander short dipole antenna printed symmetrically on the same face and loaded at both ends by Split ring resonator (SRR). The proposed design is made out of an embedded $\Pi$-match loaded at both ends with a couple of meandered arms. The comparative table shows that the volume of the proposed tag antenna is smaller than the antennas in [24] and [25] which means that our tag has the compact size. It also has the best read range and return loss compared to the other ones. In fact, the results show that the bandwidth of our RFID tag is a bit small compared to the other antennas. We can conclude that the technique of "П-match" allowed us to have the most compact structure and a good 
conjugate matching between the antenna and the chip compared to the previous presented techniques employed in [24] and [25].

Table 2. Comparison of the proposed tag and other designs.

\begin{tabular}{ccccc}
\hline Antennas & Volume $\left(\mathrm{mm}^{3}\right)$ & Return loss $(\mathrm{dB})$ & Bandwidth $(\mathrm{MHz})$ & Read range $(\mathrm{m})$ \\
\hline$[24]$ & 4412.224 & -23 & 21 & 8.3 \\
{$[25]$} & 2288 & -30 & 18 & 4.36 \\
This work & 1036.8 & -33.86 & 17.6 & 16.13 \\
\hline
\end{tabular}

\section{Conclusion}

In this paper, systematic and simple design of a novel compact antenna for passive UHF RFID tag has been introduced. To get a good conjugate matching with the chip and the proposed antenna and to ensure the compactness of our structure, the П-match network and meandered arms has been used. In order to emphasize the importance of miniaturization, a comparison between the proposed antenna with some other designed antennas in terms of volume, return loss, bandwidth, and read range is exhibited. The designed antenna has an overall volume of $54 \times 24 \times 0.8 \mathrm{~mm}^{3}$, which is smaller than those of previously reported structures. The configuration with low cost, low profile, easy fabrication, and a miniaturized size make our proposed tag suitable for UHR RFID applications.

\section{References}

[1] M.C. Tsai, C.W. Chiu, H.C. Wang, and T.-F. Wu: Inductively coupled loop antenna design for UHF RFID on-body applications. Progress n Electromagnetics Research. Vol. 143, pp. 315-330 (2013).

[2] T. Deleruyelle, P. Pannier, M. Egels, and E. Bergeret: Dual band mono-chip HF-UHF tag antenna. In 2010 IEEE Antennas and Propagation Society International Symposium. pp. 1-4, Toronto, Canada (2010).

[3] G. Marrocco: The art of UHF RFID antenna design: Impedance-matching and size-reduction techniques. IEEE Antennas Propagation Magazine, Vol. 50, pp. 66-79 (2008).

[4] G. Marrocco: Gain-optimized self-resonant meander line antennas for RFID applications. IEEE Antennas Wireless Propagation Letters. Vol. 2, pp. 302-305 (2003).

[5] P. Kamalvand, G. K. Pandey, and M. K. Meshram: RFID tag antenna for ultra and super high frequency band applications. International Journal of RF and Microwave Computer-aided Engineering, Vol. 26, No. 7, pp. 640-650 (September 2016).

[6] H. Chen, C. Tsai, C. Sim, and C. Kuo: Circularly Polarized Loop Tag Antenna for Long Reading Range RFID Applications. IEEE Antennas and Wireless Propagation Letter. Vol. 12, pp. 1460-1463 (2013).

[7] D.L. Nguyen, K.S. Paulson, and N. G. Riley: Reduced size circular polarized square microstrip antenna for 2.45GHz RFID applications. IET Microwaves, Antennas \& Propagation. Vol. 6, No.1, pp. 94-99 (2012).

[8] Y. Shi, K. Qi, and C. H. Liang: A miniaturized design of 2.45GHz RFID tag antenna. Microwave and Optical Technology Letters, Vol. 57, pp. 1905-1908 (2015).

[9] Y.Shi, C. Fang, K. Qi, and C. H. Liang: A broadband design of UHF fractal RFID tag antenna. Progress In Electromagnetics Research Letters, Vol. 58, pp. 45-51 (2016). 
[10] Y. El Hachimi, Y. Gmih, E. Makroum, and A. Farchi: A compact dual-band antenna including symmetrical slots for $2.45 / 5.8 \mathrm{GHz}$ handheld RFID Reader applications. 4th International Conference on Optimization and Applications (ICOA). Mohammedia, Morocco (April 2018).

[11] Y. Gmih, Y. El Hachimi, E. Makroum, and A. Farchi: A small printed antenna with circular slot for European UHF RFID reader devices. 4th International Conference on Optimization and Applications (ICOA). Mohammedia, Morocco (April 2018).

[12] F. Nekoogar, and F. Dowla: Ultra-Wideband Radio Frequency Identification Systems. Springer, (2011).

[13] A. Hamani, M. C. E. Yagoub, T. P. Vuong, and R. Touhami: A novel broadband antenna design for UHF RFID tags on metallic surface environments. IEEE Antennas and Wireless Propagation Letters, Vol. 16, pp. 91-94 (2017).

[14] D. K. Naji, J. S. Aziz, and R. S. Fyath: Design of Miniaturized Fractal RFID Tag Antenna with Forced Impedance Matching. International Journal of Electromagnetics and Applications. Vol. 2, no. 5, pp. 129-139 (2012).

[15] N. H. Daud, A. Ismail, A. Reda, H. Alhawari, A. Sali, and M. F. A. Rasid: Integration of Split Ring Resonators ( SRRs ) to UHF RFID Tag Antenna for Size Reduction. International Conference on Engineering Technology and Technopreneuship (ICE2T) pp. 204-208 (2014).

[16] Y. Shi, C. Fang, K. Qi, and C. H. Liang: A broadband design of UHF fractal RFID tag antenna. Progress In Electromagnetics Research Letters, Vol. 58, pp. 45-51 (2016).

[17] H.-T. Chou, J.-C. Chu, and Y.-Y. Kuo: Size reduction of patch antenna using a meander line feeding structure for RFID applications. Microwave Opt Technol Lett 56. pp. 918-920 (2014).

[18] G. Zamora, S. Zuffanelli, F. Paredes, F. Martin, and J. Bonache: Design and synthesis methodology for UHF-RFID tags based on the T-matched network. IEEE Trans. Microw. Theory Techn, Vol. 61, no. 12, pp. 4090-4098 (Dec. 2013).

[19] C.-W. Chiu, M.-C. Tsai, H.-C. Wang, and T.-F. Wu: Broadband Tmatching loop tag antenna design for on-body UHF RFID applications. Microwave and Optical Technology Letters, vol. 56, no. 5, pp. 1194-1200, (May 2014).

[20] Q. Liu, Y. F. Yu, and S. He: Capacitively loaded, inductively coupled fed loop antenna with an omnidirectional radiation pattern for UHF RFID tags. IEEE Antennas Wireless Propag. Lett. Vol. 12, pp. 1161-1164 (2013).

[21] CST Studio Suite version 2015 :http://www.cst.com/Content/Products/DS/Overview.aspx.

[22] Alien Higgs 4, www.alientechnology.com/products/ic/higgs-4/

[23] A. Ghiotto: Conception d'Antennes de Tags RFID UHF, Application à la Réalisation par Jet de Matière. Thèse de Doctorat. Institut Polytechnique de Grenoble (Novembre2008).

[24] Md. Rokunuzzaman, M. T. Islam, W. S. T. Rowe, S. Kibria, M. J .Singh, and N. Misran: Design of a Miniaturized Meandered Line Antenna for UHF RFID Tags. Public Library of Science (Plos One). Vol. 11, no. 8, pp. 1-14 (August 2016).

[25] I. Aznabet, M. Ennasar, O. El Mrabet, S. Tedjini, and M. Khalladi: Meander-Line UHF RFID Tag Antenna loaded with Split Ring Resonator. 5th International Conference on Multimedia Computing and Systems (ICMCS). Marrakech, Morocco (2016). 